\title{
RHIC Local Orbit Control and Power Supply Resolution
}

\author{
T. Satogata
}

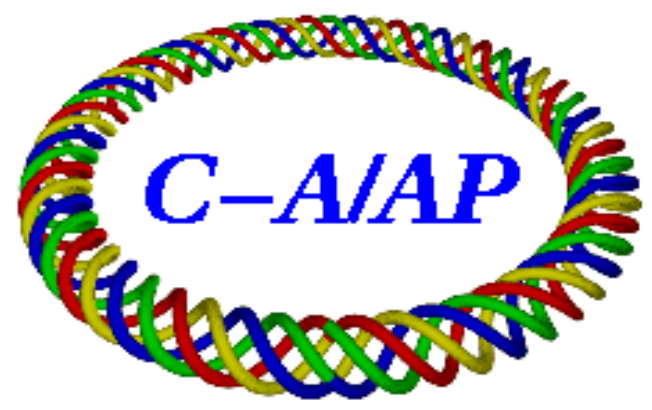

\section{Collider-Accelerator Department Brookhaven National Laboratory Upton, NY 11973}

Notice: This document has been authorized by employees of Brookhaven Science Associates, LLC under Contract No. DE-AC02-98CH10886 with the U.S. Department of Energy. The United States Government retains a nonexclusive, paid-up, irrevocable, world-wide license to publish or reproduce the published form of this document, or allow others to do so, for United States Government purposes. 


\title{
RHIC Local Orbit Control and Power Supply Resolution
}

\author{
T. Satogata
}

October 15, 2009

\begin{abstract}
Slow global orbit correction at store, running every 30-60 minutes, has been in place since RHIC Run8. This correction should include tight orbit drift tolerances at the interaction point and collimators, as these are locations where orbit drift of a few hundred microns is observable in backgrounds and luminosity. Future improvements in low beta optics will only lower these tolerances. run fy09 attempts to control the collimator orbit with local three-bumps after global orbit corrections appeared to be limited by corrector power supply resolution. This paper evaluates orbit control in the context of existing corrector power supply resolution, and makes recommendations for planned RHIC operations scenarios.
\end{abstract}

\section{Power Supplies}

The RHIC dipole corrector power supplies used for orbit steering are Suncraft bipolar $\pm 50 \mathrm{~A} 20 \mathrm{~V}$ power supplies with 12-bit controllers [1]. This gives $2^{12}=4096$ setpoints, or $24.4 \mathrm{~mA}$ resolution. Fig. 1 shows an example of measured power supply resolution for bi8-th3-ps for ramp 10866.

The measured conversion of set current to corrector strength can be found by studying a scan of dipole corrector strengths. A vernier scan was performed later in ramp 10866 which permits this comparison, as shown in Fig. 2. The MADC current readback is from the logger RHIC/PowerSupplies/Blue/BlueDipCorr8, which has better time resolution than the slow loggers in the PowerSupplies LogView branch. However, it also has an extra scaling factor of 100 in the logger file. Incorporating this, we find the measured conversion from power supply current $I$ to dipole corrector strength $\Delta x^{\prime}$ in these machine conditions is

$$
\Delta x^{\prime}[\mathrm{mrad}]=\left(0.0169 \pm 2.37 \times 10^{-4}\right) I[\mathrm{~A}]
$$

The kick strength scales inversely with $B \rho$. At the time of the vernier scan, the beam energy was $E=100 \mathrm{GeV}$, with $B \rho=334.283$ T-m. We can therefore write the dipole corrector strength dependency on current and $B \rho$ as:

$$
\Delta x^{\prime}[\mathrm{mrad}]=\left(0.0169 \pm 2.37 \times 10^{-4}\right) I[\mathrm{~A}]\left(\frac{334.283 \mathrm{~T}-\mathrm{m}}{B \rho[\mathrm{T}-\mathrm{m}]}\right)
$$

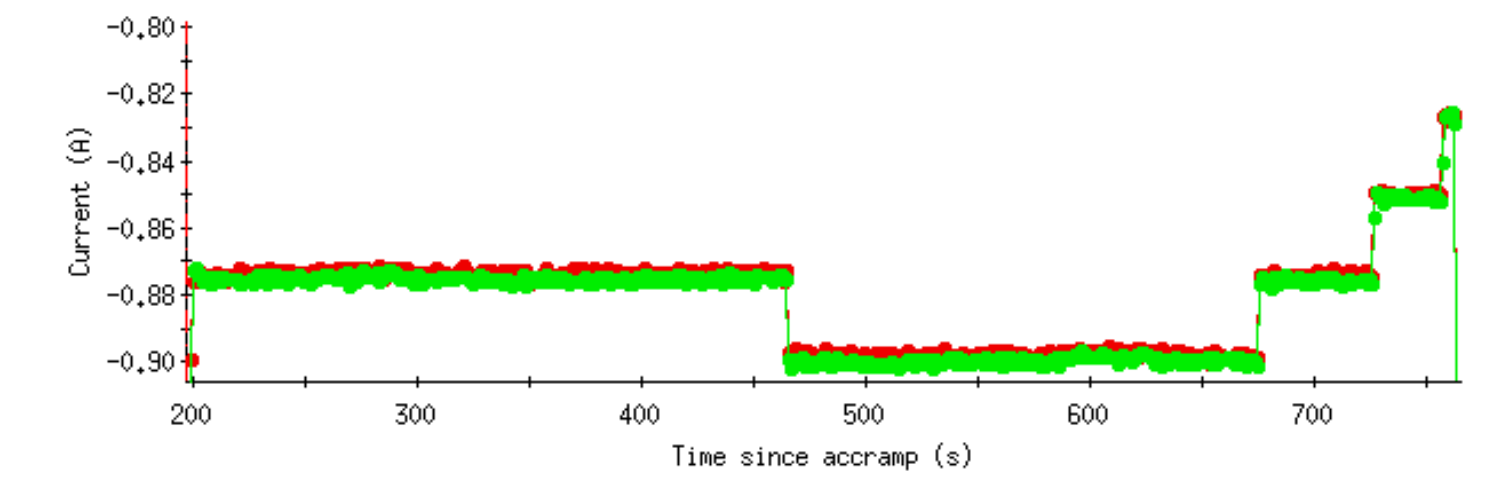

$\longrightarrow$ bi8-th3-ps_CombRamp_iref

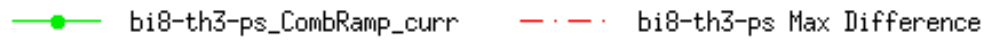

Figure 1: Reference current and readback for bi8-th3 during ramp 10866 on 5 June 2009 from pscompare. Step sizes are consistent with $24.4 \mathrm{~mA}$ resolution, and power supply regulation is good to about $1 \mathrm{~mA}$ RMS. 


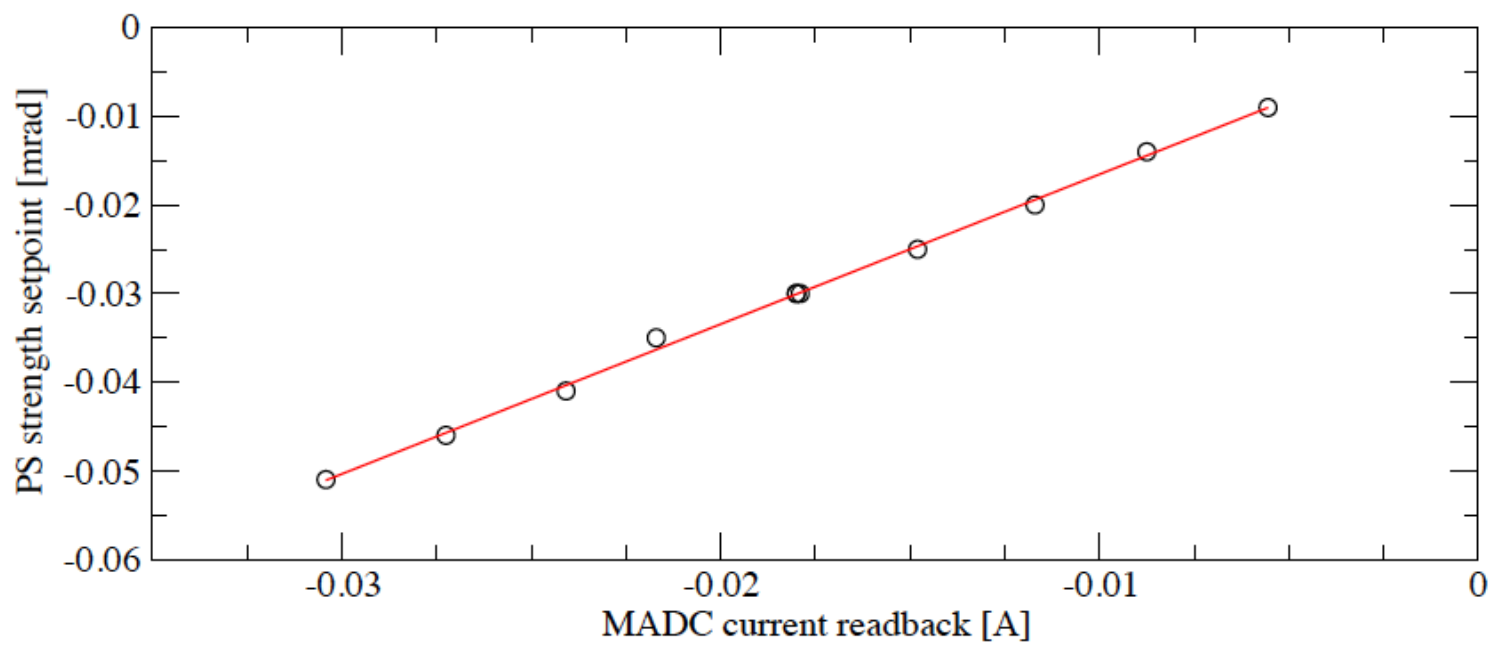

Figure 2: Power supply strength setpoint vs MADC current readback for bi8-th3-ps during a store IR8 vernier scan in ramp 10866 on 5 June 2009. Note that the MADC current readback differs from the power supply current by a factor of 100. A linear fit gives Eqn. (1).

$$
=(5.65 \pm 0.08) \frac{I[\mathrm{~A}]}{B \rho[\mathrm{T}-\mathrm{m}]}
$$

\section{Orbit Bump Resolution}

What interests us here is the bump resolution for single-bit resolution in the power supply controllers. From Eqn. (3) and the $24.4 \mathrm{~mA}$ bit resolution of the correctors,

$$
\Delta x^{\prime}[\operatorname{mrad}, \text { resolution }]=\frac{0.138 \pm 1.95 \times 10^{-3}}{B \rho[\mathrm{T}-\mathrm{m}]}
$$

For the $100 \mathrm{GeV}$ vernier scan, $\Delta x^{\prime}[\mathrm{mrad}$, resolution $]=0.4 \mu \mathrm{rad}$ while the vernier scan steps in Fig. 2 are about $5 \mu \mathrm{rad}$; resolution effects are on the order of $5 \%$ of the step size for vernier scans in this configuration.

At lower $B \rho$ the single-bit resolution is larger, and the bump control is coarser with a larger range. At higher $B \rho$ the single-bit resolution is smaller and we have finer control over bumps with a correspondingly smaller range. We are particularly interested in correction of small orbit errors at the collimators and IRs, so we consider the three- and four-bump correctors in these areas.

Define $\Delta x_{2}$ as the orbit offset at an inner corrector of a three- or four-bump. We want $\Delta x_{2}$ small to have fine-grained control over the orbit at the center of the bump. This works best when local beta functions are small and $B \rho$ is large. Conversely, one should expect challenges when local beta functions are large and $B \rho$ is relatively small.

Table 1 lists some RHIC conditions where local bump control is most likely resolution-limited. The collimator/IR conditions use design optics around the IR 8 collimators and IR steering bumps. These have almost the same constraints because they are dominated by the same Q3/4 dipole correctors in their bumps. This table shows that high-energy RHIC conditions benefit from high $B \rho$, even when low- $\beta$ optics are in place that raise local $\beta_{\max }$ in the triplets and make bumps correspondingly more sensitive.

Table 1 also lists the corrector resolutions as calculated from Eqn. (4), the bit-resolution three- or fourbump amplitude, and closed bump coefficients as calculated from MAD design optics. The smallest bump coefficients have the highest sensitivity, so the bit-resolution bump amplitude is the corrector resolution divided by the lowest (or most sensitive) bump coefficient. For example, the $100 \mathrm{GeV}$ pp horizontal collimator bit-resolution bump amplitude is $(0.41 \mu \mathrm{rad}) /(3.1 \mu \mathrm{rad} / \mathrm{mm})=0.132 \mathrm{~mm}$.

Some entries, such as this $100 \mathrm{GeV}$ pp horizontal collimator three-bump, are dominated by high beta at edge correctors; attempts to create bumps smaller than this bit resolution will not be closed and will leak around the ring. Other entries, such as the $100 \mathrm{GeV}$ pp IR horizontal four-bump and the low energy vertical arc three-bumps, have small coefficients within the bump. These act more like three- or two-bumps, and the sensitive corrector can be set to zero strength for reasonably bump control. Entries listed in bold in Table 1 use this constraint, and usually gain a factor of 5-10 in bit-resolution bump amplitude. 
Table 1: RHIC conditions where local bump control is most severely limited by corrector power supply resolution, according to Eqn. (4). The bump coefficients are corrector strengths in mrad that give a $1 \mathrm{~mm}$ bump at the specified location. Boldface entries are described in the text. The near-integer pp conditions are at injection rigidity with tunes of $(28.92,29.91)$.

\begin{tabular}{|c|c|c|c|c|c|}
\hline Machine conditions & $\begin{array}{l}\beta^{\star} \\
{[\mathrm{m}]}\end{array}$ & $\begin{array}{c}B \rho \\
{[\mathrm{T}-\mathrm{m}]}\end{array}$ & $\begin{array}{c}\text { Corr resolution } \\
{[\mu \mathrm{rad}]}\end{array}$ & $\begin{array}{l}\text { bump amplitude } \\
\text { [mm, resolution] }\end{array}$ & $\begin{array}{l}\text { bump coefficients } \\
{[\mu \mathrm{rad} / \mathrm{mm}]}\end{array}$ \\
\hline $100 \mathrm{GeV}$ pp collimator $\mathrm{H}$ & \multirow{4}{*}{0.7} & \multirow{4}{*}{333.5} & \multirow{4}{*}{0.41} & 0.132 & $8.1 / 43.3 / 3.1$ \\
\hline $100 \mathrm{GeV}$ pp collimator $\mathrm{V}$ & & & & 0.019 & $27.6 /-207.6 / 21.6$ \\
\hline $100 \mathrm{GeV}$ pp IR H & & & & $0.084 / \mathbf{0 . 0 1 6}$ & $27.2 / \mathbf{- 4 . 9} / 40.3 / 25.7$ \\
\hline $100 \mathrm{GeV}$ pp IR V & & & & 0.109 & $8.3 / 3.8 /-14.5 / 7.5$ \\
\hline $100 \mathrm{GeV} / \mathrm{u} \mathrm{Au}$ collimator $\mathrm{H}$ & \multirow[t]{4}{*}{0.5} & \multirow[t]{4}{*}{839.5} & \multirow[t]{4}{*}{0.16} & 0.063 & $7.7 / 45.8 / 2.7$ \\
\hline $100 \mathrm{GeV} / \mathrm{u} \mathrm{Au}$ collimator $\mathrm{V}$ & & & & 0.006 & $29.1 /-242.4 / 24.0$ \\
\hline $100 \mathrm{GeV} / \mathrm{u} \mathrm{Au} \mathrm{IR} \mathrm{H}$ & & & & $0.034 / \mathbf{0 . 0 0 6}$ & $27.9 / \mathbf{- 4 . 8} / 43.1 / 26.9$ \\
\hline $100 \mathrm{GeV} / \mathrm{u} \mathrm{Au} \mathrm{IR} \mathrm{V}$ & & & & 0.047 & $8.0 / 3.5 /-14.7 / 7.1$ \\
\hline inj pp near-int collimator $\mathrm{H}$ & \multirow[t]{4}{*}{10} & \multirow[t]{4}{*}{79.37} & \multirow[t]{4}{*}{1.74} & 0.261 & $11.6 / 19.7 / 6.7$ \\
\hline inj pp near-int collimator $\mathrm{V}$ & & & & 0.128 & $21.7 /-60.2 / 13.6$ \\
\hline inj pp near-int IR H & & & & 0.247 & $21.8 /-7.0 / 26.2 / 17.3$ \\
\hline inj pp near-int IR V & & & & 0.244 & $11.6 / 7.1 /-11.5 / 9.3$ \\
\hline $7.7 \mathrm{GeV} / \mathrm{u} \mathrm{Au}$ collimator $\mathrm{H}$ & \multirow[t]{6}{*}{10} & \multirow[t]{6}{*}{31.1} & \multirow[t]{6}{*}{4.44} & 0.62 & $11.9 / 15.6 / 7.1$ \\
\hline $7.7 \mathrm{GeV} / \mathrm{u} \mathrm{Au}$ collimator $\mathrm{V}$ & & & & 0.33 & $21.6 /-55.2 / 13.4$ \\
\hline $7.7 \mathrm{GeV} / \mathrm{u}$ Au IR H & & & & 0.60 & $21.5 /-7.4 / 26.2 / 16.7$ \\
\hline $7.7 \mathrm{GeV} / \mathrm{u} \mathrm{Au} \mathrm{IR} \mathrm{V}$ & & & & 0.62 & $11.9 / 7.1 /-12.5 / 9.2$ \\
\hline $7.7 \mathrm{GeV} / \mathrm{u} \mathrm{Au}$ arc $\mathrm{H}$ & & & & 0.83 & $21.8 / 5.4 / 21.8$ \\
\hline $7.7 \mathrm{GeV} / \mathrm{u} \mathrm{Au} \operatorname{arc} \mathrm{V}$ & & & & $2.22 / \mathbf{0 . 2 0}$ & $22.1 / \mathbf{2 . 0} / 22.1$ \\
\hline $5.0 \mathrm{GeV} / \mathrm{u} \mathrm{Au}$ collimator $\mathrm{H}$ & \multirow[t]{6}{*}{8} & \multirow[t]{6}{*}{19.3} & \multirow[t]{6}{*}{7.15} & 1.06 & $11.6 / 18.7 / 6.8$ \\
\hline $5.0 \mathrm{GeV} / \mathrm{u} \mathrm{Au}$ collimator $\mathrm{V}$ & & & & 0.53 & $21.7 /-59.8 / 13.6$ \\
\hline $5.0 \mathrm{GeV} / \mathrm{u} \mathrm{Au}$ IR H & & & & 0.99 & $21.7 /-7.2 / 26.2 / 17.2$ \\
\hline $5.0 \mathrm{GeV} / \mathrm{u} \mathrm{Au}$ IR V & & & & 1.00 & $11.6 / 7.2 /-11.9 / 9.2$ \\
\hline $5.0 \mathrm{GeV} / \mathrm{u} \mathrm{Au}$ arc $\mathrm{H}$ & & & & 1.35 & $21.8 / 5.3 / 21.8$ \\
\hline $5.0 \mathrm{GeV} / \mathrm{u} \mathrm{Au}$ arc $\mathrm{V}$ & & & & $3.97 / \mathbf{0 . 3 2}$ & $22.1 / \mathbf{1 . 8} / 22.1$ \\
\hline
\end{tabular}

Note that Table 1 bit-resolution bump amplitudes are in the center of the given three- or four-bump. The resolution of bump control at the collimators may be slightly better or worse than the listed resolution depending on the optics configuration, but it is likely no worse those listed here.

\section{Global Orbit Correction}

The RMS orbit distortion in a synchrotron ring at a location with beta function $\beta_{0}$ from an ensemble of $N$ steering errors of RMS value $\theta_{\mathrm{r} m s}$ at average beta function $\bar{\beta}$ is [2]

$$
\Delta x_{\mathrm{rms}}=\frac{\left(\beta_{0} \bar{\beta}\right)^{1 / 2}}{2 \sqrt{2}|\sin \pi Q|} N^{1 / 2} \theta_{\mathrm{rms}}
$$

At measurement $\beta_{\max }$ locations in the arc FODO cells, $\beta_{0} \approx 50 \mathrm{~m}$. We have about $N \approx 120$ dipole correctors in each plane, with $\bar{\beta} \approx 80 \mathrm{~m}$. For $N$ random single-bit errors, $\theta_{\text {rms }}=\Delta x^{\prime}[\mathrm{mrad}$, resolution $] / \sqrt{2}$. With these numbers, we have

$$
\Delta x_{\mathrm{rms}}[\mathrm{mm}] \approx \frac{0.17}{|\sin \pi Q|} \Delta x^{\prime}[\mu \mathrm{rad}, \text { resolution }]
$$

Eqn. (6) is only a rough estimate, as it assumes that all dipole corrector power supplies change by one bit in an uncorrelated way. Power supply resolution and its effects on correlated corrector strength changes during orbit correction should be investigated in more detail. Table 2 indicates that orbit corrections at the 
Table 2: RHIC conditions where local bump control is most severely limited by corrector power supply resolution, according to Eqn. (4). The bump coefficients are corrector strengths in mrad that give a $1 \mathrm{~mm}$ bump at the specified location. Boldface entries are described in the text. The near-integer pp conditions are at injection rigidity with tunes of $(28.92,29.91)$.

\begin{tabular}{lccccc}
\hline Machine conditions & $\begin{array}{c}\beta^{\star} \\
{[\mathrm{m}]}\end{array}$ & $\begin{array}{c}B \rho \\
{[\mathrm{T}-\mathrm{m}]}\end{array}$ & $\begin{array}{c}\text { Corr resolution } \\
{[\mu \mathrm{rad}]}\end{array}$ & $\begin{array}{c}\text { Fractional } \\
\text { Tune } Q\end{array}$ & $\begin{array}{c}\Delta x_{\mathrm{rms}} \\
{[\mathrm{mm}, \text { from }(6)]}\end{array}$ \\
\hline 100 GeV pp & 0.7 & 333.5 & 0.41 & 0.69 & 0.084 \\
$100 \mathrm{GeV} / \mathrm{u} \mathrm{Au}$ & 0.5 & 839.5 & 0.16 & 0.23 & 0.041 \\
inj pp near-int & 10 & 79.37 & 1.74 & 0.92 & 1.189 \\
inj pp near-int & 10 & 79.37 & 1.74 & 0.95 & 1.891 \\
inj pp near-int & 10 & 79.37 & 1.74 & 0.96 & 2.360 \\
$7.7 \mathrm{GeV} / \mathrm{u} \mathrm{Au}$ & 10 & 31.1 & 4.44 & 0.11 & 2.228 \\
$5.0 \mathrm{GeV} / \mathrm{u} \mathrm{Au}$ & 8 & 19.3 & 7.15 & 0.11 & 3.588 \\
\hline
\end{tabular}

level of $1 \mathrm{~mm}$ rms are challenging for RHIC near-integer and low energy operations, and also require further evaluation.

\section{Summary and Conclusions}

RHIC dipole corrector power supplies provide a kick $\Delta x^{\prime}$ that depends on the dipole corrector current $I$ and machine rigidity $B \rho$ :

$$
\Delta x^{\prime}[\mathrm{mrad}]=(5.65 \pm 0.08) \frac{I[\mathrm{~A}]}{B \rho[\mathrm{T}-\mathrm{m}]}
$$

The 12-bit controllers for these power supplies give single-bit kick resolution of

$$
\Delta x^{\prime}[\operatorname{mrad}, \text { resolution }]=\frac{0.138 \pm 1.95 \times 10^{-3}}{B \rho[\mathrm{T}-\mathrm{m}]}
$$

We have calculated minimum bump strengths based on this corrector resolution for several machine conditions where challenges are expected with existing RHIC dipole corrector resolution. These include threebumps at the collimators, four-bumps for low-beta IR beam steering, and arc three-bumps for low energy operations where we expect to encounter challenges with RHIC corrector resolution. These values are listed in Table 1. We also estimate the rms orbit deviation for random one-bit changes at all dipole correctors as a possible bound on global orbit correction resolution, including near-integer pp operations at injection energy. These estimates are listed in Table 2.

\subsection{Observations}

- The combination of low-beta optics, optics configuration (large beta functions at the edges of the surrounding three-bump), and low $B \rho$ made collimator horizontal orbit control during run_fy09 100 $\mathrm{GeV}$ pp operations difficult at precisions lower than $100 \mu \mathrm{m}$. There was likely no such limit in the vertical collimator control.

- The combination of low-beta optics and low $B \rho$ also made vertical orbit control at the PHENIX IR difficult at precisions lower than $100 \mu \mathrm{m}$. This should be compared to experience with PHENIX vernier scans during that run.

- The previous two limitations benefit from higher $B \rho$ for planned run fy10 $100 \mathrm{GeV} / \mathrm{u} \mathrm{Au}$ operations, though orbit control at precisions below $50 \mu \mathrm{m}$ will still be corrector limited.

- Near-integer polarized proton operations is most limited by corrector resolution near injection with small $B \rho$. In these conditions, global orbit corrections may be resolution limited to about 1-2 mm rms depending on proximity to integer tune. Global orbit correction limitations from corrector resolution requires further study, as run_fy09 near-integer orbit corrections converged to $0.3 \mathrm{~mm}$ rms after several iterations, but did not improve beyond that limit with further iterations [3]. 
- Anticipated run_fy10 low energy operations with $7.7 \mathrm{GeV} / \mathrm{u}$ Au may be limited by dipole corrector resolutions, though the large beam size will likely diminish its impact. However, global and local orbit correction should be revisited, since resolution-limited three-bumps in the arcs are already about 0.5 $\mathrm{mm}$ and global orbit corrections may be resolution limited to about $2 \mathrm{~mm} \mathrm{rms}$.

- Anticipated future low energy operations with $5.0 \mathrm{GeV} / \mathrm{u} \mathrm{Au}$ will likely be compromised by dipole corrector resolution with the existing configuration.

\subsection{Recommendations}

- Develop alternate methods, such as BPM weighting in global orbit corrections, to improve orbit control around collimators and IRs during routine operations. Existing three-bump local control methods are resolution limited for horizontal orbit control at the collimators at the level of 50-150 $\mu \mathrm{m}$.

- Simulate and evaluate corrector resolution effects on global orbit correction convergence in the cases of near-integer pp operations at injection energy, and low energy Au operations.

- The 12-bit DACs are the limiting factor in dipole corrector control in RHIC. Observed power supply regulation is about a factor of 20 below the DAC resolution. Changing dipole corrector control DACs from 12-bit to 16-bit, as was already done with RHIC sextupoles, will improve corrector control down to about the level of observed power supply regulation and satisfy existing and future program needs for fine orbit control. Correctors in the region of the collimators and low-beta IRs are the highest priority for this upgrade, and should serve as a test bed for global implementation.

\section{References}

[1] OPSYB1 RHICgddb..ps_vista power supply database.

[2] Edwards and Syphers, "An Introduction to the Physics of High Energy Accelerators", Wiley (1993), p. 103.

[3] V. Ptitsyn, private communication. 